\title{
Comunicación corporativa y branded content en Facebook: un estudio de las cuentas oficiales de las universidades peruanas
}

\author{
Corporate communications and branded content on Facebook: \\ A study of the official accounts of Peruvian universities
}

\author{
Atarama-Rojas, T. y Vega-Foelsche, D. ${ }^{1}$ \\ Recibido: 23-04-2019 - Aceptado: 10-01-2020 \\ DOI: https://doi.org/10.26441/RC19.1-2020-A3
}

\begin{abstract}
RESUMEN: Esta investigación explora el uso de Facebook como un canal de comunicación corporativa de las universidades peruanas y persigue el objetivo de reconocer cuáles son los elementos comunicativos que generan mayor interacción. Para alcanzar este objetivo, se realiza un análisis de contenido que permite detectar las acciones comunicativas, los formatos, las temáticas y los vínculos emocionales que propician una mayor participación de los públicos en esta red social. Además, se complementa esta metodología con un análisis cualitativo de las publicaciones más relevantes. Los resultados de la investigación muestran que los contenidos más valorados por el público son aquellos que buscan educar e inspirar, a través de formatos audiovisuales, con los resultados de investigación e innovación científica, apelando a un sentimiento de orgullo de la comunidad.
\end{abstract}

Palabras claves: branded content; redes sociales; facebook; universidades; comunicación digital; marketing de contenidos.

ABSTRACT: This research explores the use of Facebook as a corporate communications channel of Peruvian universities. The study aims to find out which communication elements generate the greatest interaction. To this end, content analysis is carried out, which allows to detect communicative actions, formats, themes and emotional links that lead to greater participation of audiences in this social network. This methodology is complemented by a qualitative analysis of the most relevant posts. Results show that content valued most by the public seeks to educate and inspire with the results of research and scientific innovation and through audiovisual formats, appealing to a sense of pride of the community.

Keywords: branded content; social networks; facebook; universities digital communication; content marketing.

\footnotetext{
${ }^{1}$ Tomás Atarama-Rojas es Máster en Creación de Guiones Audiovisuales por la Universidad Internacional de La Rioja, Magíster Académico en Comunicación y candidato a Doctor en Comunicación por la Universidad de los Andes. Es Profesor de Guion Audiovisual en la Universidad de Piura. tomas.atarama@udep.edu.pe, https://orcid. org/0000-0002-4430-3391

Diego Vega-Foelsche es Licenciado en Comunicación por la Universidad de Piura, cursó el Diplomado en Marketing Digital Estratégico y se desempeña como Gestor de Comunicación en Social Networks, responsable de la estrategia de redes sociales para los públicos internos y externos de la Universidad de Piura. diego.vega@udep. edu.pe, https://orcid.org/0000-0002-9166-5053
} 


\section{Introducción}

Los usos de las redes sociales para la gestión de la comunicación de las organizaciones han ido en aumento, debido a que generan una mayor cercanía con sus públicos de interés de modo global, rápido, directo y a un menor coste. Según Mazza y Palermo (2019), en la actualidad, alrededor del $84 \%$ de las organizaciones utilizan las redes sociales para comunicarse con sus stakeholders.

Ante la búsqueda de formas de comunicación que conecten con los usuarios en redes sociales, se ha popularizado el marketing de contenidos, que consiste en atraer al usuario con publicaciones de interés que lo induzcan a realizar una determinada acción deseada por la institución, pero sin que el usuario se sienta condicionado. Por el contrario, debe sentir que está tomando una decisión con total libertad a partir de lo que le interesa, y no de lo que una organización le quiere vender (Coto y Borowiecka, 2014). Estas estrategias de contenidos responden al planteamiento de growth hacking, que consiste en compartir contenidos que maximicen la visibilidad de la marca y el crecimiento de su comunidad de seguidores en redes sociales (Coll, 2019).

Una de ellas es el branded content, que consiste en producir contenidos que el usuario desea consumir por su significado en términos de utilidad y entretenimiento (Moyano, Baños y Ramírez, 2016). Este modelo de comunicación en el que se enmarca el branded content en redes sociales es aprovechado con mayor frecuencia por marcas que han nacido en la era digital y que se caracterizan por procesos horizontales que buscan una interconexión y experiencia en el relacionamiento con sus públicos a una escala global. Aplicar este modelo de comunicación a organizaciones tradicionales y con estructuras verticales, como las universidades, es un reto mayor. No obstante, las universidades tienen en común, por su naturaleza, el rol de formar profesionales preparados, investigar las problemáticas del entorno y orientar el desarrollo de la sociedad a través del conocimiento (Villegas, 2018), por lo que cuentan con un capital intelectual que puede integrarse en estrategias de branded content que aporten utilidad al usuario.

Este estudio persigue el objetivo de reconocer cuáles son los elementos comunicativos que generan mayor interacción, para esto explora de qué modo emplean las universidades peruanas la red social de Facebook y la composición de los contenidos que resultan más valiosos para sus usuarios. Así, se plantea la siguiente hipótesis: En las cuentas oficiales de Facebook de las mejores universidades del Perú, según la Superintendencia Nacional de Educación Superior Universitaria (Sunedu) ${ }^{2}$, las publicaciones con mayor interacción son aquellas que aportan un contenido que educa e inspira a partir del capital intelectual con el que cuentan las instituciones universitarias (investigaciones), usando formatos audiovisuales y apelando al orgullo que puede despertar en su comunidad dichas publicaciones.

Como metodología de investigación, se ha desarrollado una propuesta que contempla herramientas de análisis cuantitativo y cualitativo. Esta metodología ha permitido, primero, conocer qué elementos comunicativos responden mejor a los intereses y preferencias de los usuarios, a través del análisis de cada elemento de manera aislada. Segundo, se ha procedido a un análisis cualitativo de las diez mejores publicaciones de cada universidad con la finalidad de contrastar el uso e incidencia de los elementos comunicativos. Se ha recogido un total de 2495 publicaciones realizadas en el periodo académico 2018-I.

A través de las reflexiones sobre las redes sociales y el branded content, se propone el planteamiento de una comunicación corporativa que concibe al contenido como motor de la nueva economía

\footnotetext{
${ }^{2}$ La Sunedu es un organismo público técnico especializado, adscrito al Ministerio de Educación del Perú, que se encarga del licenciamiento de las universidades y de verificar el cumplimiento de las condiciones básicas de calidad en el sistema de educación superior en el Perú (Sunedu, 2019).
} 
digital, que es evaluado en función al nivel de interacción de sus públicos, en la medida que genera una mayor visibilidad de marca y el crecimiento de la comunidad de seguidores en red. Esta investigación espera contribuir al ejercicio profesional de las direcciones y departamentos de comunicación de las universidades que, en los últimos años, vienen asumiendo la gestión de la marca a partir de sus signos distintivos y de la coordinación estratégica de sus departamentos.

\section{Marco teórico}

\subsection{Naturaleza y características de las redes sociales: un espacio al servicio del usuario}

Una red social es una estructura de comunicación conformada por personas que tienen intereses y características en común que deciden voluntariamente formar parte de una comunidad (Critikián, 2017). Por ello, cuando nos referimos a las redes sociales como canal de comunicación, nos referimos a plataformas tecnológicas al servicio de lo que la persona viene haciendo desde siempre: relacionarse para compartir e intercambiar sus ideas y sentimientos como ser social que es (Mosquera y Bartolomé, 2014).

Ante lo expuesto, una red social no consiste solo en un canal de acceso a la información, sino que consiste en una plataforma de relacionamiento entre personas y comunidades (Coto y Borowiecka, 2014). En función de cómo se tejen las redes de cooperación, las plataformas se clasifican como horizontales o generalistas (Facebook, Instagram, Twitter), y como verticales o especializadas (LinkedIn, Soundcloud, Flickr) cuando los usuarios interactúan en comunidades segmentadas por ámbito geográfico, temático y/o sociodemográfico (Rodríguez-Fernández, Sánchez-Amboage y Juanatey-Boga, 2019).

Partiendo del análisis de Critikián (2017) y Sánchez (2017), a continuación, se presentan las características esenciales de las redes sociales. Estas se recogen desde un doble enfoque que considera, por un lado, a las redes sociales como plataformas tecnológicas y, por otro, el modo en que las personas las utilizan. Estas características también son recogidas por la Interactive American Bureau (2018), principal organismo a nivel global que representa a la industria de la publicidad online en el mundo.

Conectividad: Las personas participan en las redes sociales a través de conexiones formadas en virtud de las características o intereses que tienen en común.

Interactividad: Las personas interactúan con las publicaciones realizadas por sus amigos y comunidades a las que pertenecen en sus redes.

Identidad: Las personas crean una cuenta y la configuran en base a sus datos personales, sus preferencias, pasatiempos, ocupación y otros elementos que guardan relación con el desarrollo de sus vidas.

Instantaneidad: Las interacciones pueden realizarse de forma inmediata, permitiendo un intercambio fluido y constante en la red.

Multimedialidad: Las redes sociales admiten variedad de contenidos textuales, visuales y audiovisuales para compartir en las plataformas.

Coparticipación: La participación e intercambio de contenidos generan una inteligencia colectiva, por lo que un contenido solo tiene relevancia en la medida en que otros usuarios participan de él.

Las redes sociales son plataformas que, en función a su naturaleza, tienen en común proveer un espacio relacional en el que sus usuarios pueden producir, recibir y poner en contacto informa- 
ción, opiniones, imágenes, o cualquier otro contenido comunicativo que les signifique valor. Así, comprender la naturaleza de las redes sociales significa concebirlas como un espacio o plataforma comunicativa de carácter bidireccional e interactivo dónde la conversación se genera en virtud del compartir y no de la sola presencia pasiva de usuarios (Rodrigo, García y Martín, 2011).

\subsection{Las organizaciones en redes sociales: el reto de las universidades}

Antes de las redes sociales, la presencia de una organización en los medios se clasificaba en propios (revistas, boletines de noticias, página web), medios pagados (publicidad en diarios, televisión, radio) y medios ganados (menciones, publicity). No obstante, hoy se debe entender que existen otros canales con un modelo comunicativo distinto al de los medios tradicionales, que son las redes sociales, y se les debe concebir en la clasificación como medios compartidos, donde los contenidos son creados en coparticipación con comunidades de usuarios en línea. Los medios pagados han dejado de tener la hegemonía en los presupuestos de comunicación; ha aumentado la participación de marca en aquellos espacios que ayudan a generar compromiso con el usuario y permiten crear experiencias que atraigan a nuevos usuarios para la construcción de audiencias propias (Del Pino y Castelló-Martínez, 2015).

Para las organizaciones que han nacido en la era digital, es más fácil adaptarse a las exigencias del entorno con estructuras horizontales y procesos gestionados para garantizar una eficiente comunicación y experiencia de sus públicos, internos y externos. Sin embargo, resulta un reto mayor para las instituciones tradicionales y burocratizadas, debido a que la rigidez de sus estructuras no les permite el desarrollo de procesos ajustados a las exigencias de conectividad e inmediatez que suponen las nuevas plataformas de comunicación, como el caso de las universidades (Reina, Fernández y Noguer, 2012).

Los nuevos medios compartidos de comunicación, como las redes sociales, requieren que las universidades consideren dos aspectos: establecer metas claras que se desean alcanzar y diseñar de qué manera se van a lograr con la participación de los públicos, internos y externos (Mazza y Palermo, 2019). Como explica Opresnik (2018), la participación de las marcas en el entorno digital requiere el diseño de una metodología SMART que involucre a toda la organización: los objetivos que se desean alcanzar deben ser específicos (Specific), cuantificables (Measurable), acorde a los objetivos de la organización (Achievable), realistas en base a los recursos (Realistic) y estructurados en un marco de tiempo realizable (Time).

Una vez que la organización ha establecido los objetivos, debe realizar una auditoría de las redes sociales en las que desea tener presencia. Este paso le da a la organización información valiosa sobre las plataformas en las que están presentes sus públicos y de qué modo puede relacionarse con ellos entendiendo sus hábitos, preferencias y estilos (Opresnik, 2018). La auditoría implica observar a sus miembros, aprender de su lenguaje y modos de comportamiento. En palabras de TúñezLópez, Valarezo-González y Punín-Larrea (2019), implica seguir una regla sociológica básica de todo miembro que quiere pertenecer a un grupo: observar y aprender a ser uno más.

Aprender a ser uno más no significa que las universidades renuncien a sus signos distintivos, sino que, a partir de ellos, sean capaces de aportar valor a los demás, en función de las formas y preferencias de los usuarios a la hora de interactuar en estos espacios. Como señalan Rivera-Rogel et al. (2019), el diseño organizacional y los procesos de comunicación interna de las universidades deben favorecer el trabajo de las direcciones de comunicación que trabajan en la construcción y gestión de la comunidad en línea a través de los contenidos que generan las universidades por sus propias características (educación, investigación y extensión) y que pueden resultar valiosas para sus públicos. 
Los servicios que brindan las universidades son complejos porque implican esencialmente la gestión de elementos intangibles, por ejemplo, la educación como servicio es imposible de almacenar y visualizar, al mismo tiempo, es posible solo en colaboración con el usuario (García-Feijoo, Eyzaguirre y Yabar, 2016). Los esfuerzos en el desarrollo de la comunicación corporativa de una universidad conllevan atender a públicos dispersos, "donde cada grupo tiene afinidades diferentes y busca información que es de su interés” (Rivera-Rogel, Yaguache, Velásquez y Paladines, 2019, p. 507).

El proceso de comunicación de las universidades también es complicado por la amplia dimensión de las acciones y responsabilidades de la organización.

Las universidades tienen un triple papel como centros del conocimiento. Tienen el deber de enseñar, esto es, transmitir desde sus departamentos, y sus centros, el proceso académico, formando con ello a profesionales preparados. También, tienen la obligación de buscar, de investigar, de conocer y dar respuesta al entorno que les rodea, a través de la investigación básica y aplicada. Por último, es su función fundamental, la de transferir esos conocimientos más allá de las aulas, llegar con su saber y sus hallazgos científicos a la sociedad, en general. Ahora bien, a estas tres funciones se une otra misión fundamental como es la de difusión. (Parejo, Martín-Pena, y Pinto-Zúñiga, 2016, p. 523)

Las redes sociales proporcionan un sistema de comunicación ágil y de apertura entre las universidades y sus públicos para poner en valor la contribución del quehacer institucional en el beneficio de la comunidad. Para el caso de Twitter, por ejemplo, Gómez y Paniagua (2014, p. 683) precisan que es "una herramienta cada vez más importante en las tareas de difusión de información corporativa". Y como señalan Rivera-Rogel et al. (2019, p. 506): "La Universidad como Institución de Educación Superior tiene claro que debe aprovechar el uso de las redes sociales para ofrecer una comunicación más ágil y cercana de acuerdo con las demandas actuales".

En este sentido, contribuir con comunicación práctica que oriente a la comunidad en temas de desarrollo y bienestar social, a partir de su capital intelectual, genera un impacto positivo en los intangibles de la organización, especialmente en su reputación (Atarama y Cortéz, 2015). Para ello "se hace imprescindible comunicar la importancia del quehacer universitario e implicar a la sociedad, incluso a la ajena a la comunidad universitaria, en el impulso y apoyo a la educación superior y a la investigación" (Parejo et al., 2016, p. 525).

\subsection{Branded content en redes sociales}

López-Rúa (2016) señala que las personas utilizan las redes sociales con la finalidad de satisfacer determinadas necesidades funcionales como obtener información, realizar transacciones, hacer consultas, revisar noticias de interés o simplemente para entretenerse. Serrano-Puche (2016) agrega que, además, los usuarios interactúan con los contenidos por intereses de carácter psicológico y sociológico, relacionados, principalmente, con la configuración de la identidad de la persona y del entorno. Según Koivula, Keipi, Koiranen, y Räsänen (2018), la participación individual y en comunidad de las personas corresponde a las necesidades de autorrealización y seguridad que experimenta la persona por naturaleza.

Alrededor de los contenidos atractivos, útiles e interesantes para los usuarios, se desarrollan conversaciones (Coto y Borowiecka, 2014). Se debe entender el concepto de conversación como movimiento de participación en red capaz de generar visibilidad y crecimiento de determinadas realidades en redes sociales y plataformas digitales; es a través de la construcción de estas relaciones de conversación con los públicos que la "identidad comunitaria se fortalece y estabiliza" (Mazza y Palermo, 2019, p. 385). En este sentido, las organizaciones se centran hoy en el desarrollo estratégico del contenido como motor de la nueva economía digital, que es medido a través del indicador 
engagement o nivel de interacción y que es gestionado con el objetivo de potenciar el posicionamiento de marca y el aumento de su comunidad de seguidores.

Así, en un contexto en el cual las marcas han comprendido que las personas utilizan sus redes sociales para satisfacer el deseo de consumir contenidos útiles y entretenidos, el contenido se ha convertido en una moneda de intercambio capaz de lograr grandes flujos de participación (liquid content) y comunicación con el usuario (Túñez-López et al., 2019), creando conversaciones en redes sociales sobre un aspecto de la realidad que la comunidad quiere recibir y la organización desea compartir (Del Pino y Castelló-Martínez, 2015). En suma, los usuarios esperan recibir contenidos que les aporten valor. Las temáticas y preferencias dependerán de múltiples factores relacionados con los intereses del usuario y el tipo de contenido que los usuarios comparten en determinada red (Mazza y Palermo, 2019).

En este sentido, no existe una temática estándar, ni un modo de hacer contenidos que garantice la participación interactiva de los usuarios. Como señalan Coto y Borowiecka (2014), las personas no esperan recibir contenidos "impecables", sino que sean desarrollados con empatía y a la medida de sus gustos e intereses (Atarama-Rojas y Sánchez, 2018; Túñez-López et al., 2019). Con ello, crece una tendencia que responde a un cambio de actitud en la comunicación de marca conocida como rock y love (Sáez, Núñez y Cubell, 2016). Con el término de rock, se hace referencia a que los contenidos deben sorprender a los usuarios y, con love, que no deben ser intrusivos, sino queridos por las personas. Este modelo de comunicación de marca responde a lo que Ramos (2006) llama comunicación pull, buscada por los consumidores, y no forzada, impulsada por las empresas (push).

En línea con ese modelo, se ha popularizado el marketing de contenidos. En el marketing de contenidos, el producto comunicativo, en lugar de interrumpir al usuario, lo hace participar de una buena experiencia (Niño, González y Valderrama, 2016). Esta estrategia se fundamenta en la producción de contenidos capaces de captar el interés y la atención de los clientes o posibles consumidores, en línea o fuera de ella, con fines comerciales, pero en formatos no tradicionales, basados en el entretenimiento o utilidad de la información (Dans, 2017). Esta fórmula de comunicación se ha potenciado con el internet porque el usuario libremente accede a la red y siente que nadie le está intentando vender nada (López-Rúa, 2016).

Contrario a perseguir un objetivo tangible de manera directa, el branded content se centra en generar compromiso y cercanía con los usuarios y responde a la necesidad actual que tienen las organizaciones por generar con sus públicos lealtad, confianza y una valoración positiva que genere una receptividad colaborativa para el desarrollo de sus objetivos; esto ha cobrado mayor fuerza con al auge de las redes sociales (Castello-Martínez et al., 2014; Curiel y Ferreira, 2017).

Aún existe confusión en los términos, tanto en la práctica como en la literatura académica (Coll, 2019), y se confunde el concepto de marketing de contenidos con el de content marketing, debido a que se traduce de manera literal al castellano (López-Rúa, 2016). El concepto content marketing se refiere a aquellos contenidos de carácter útil o formativo que una marca produce para atraer el interés de sus públicos en una estrategia de branded content, por ejemplo, un tutorial o una infografía de interés. También, existe el branded entertainment, una variante del branded content que implica entregar entretenimiento al usuario; por ejemplo, produciendo un corto o un videojuego (Moyano et al., 2016). El branded content no comprende entonces una acción de venta, sino de relación; dirigida a despertar en el público objetivo un interés para que sea este quien libremente decida conocer más de la marca y del valor que esta le puede ofrecer (Del Pino y Castelló-Martínez, 2015; Ashley y Tuten, 2015).

Contrario a la idea de que el branded content es una publicidad disfrazada (Pineda, 2009), los usuarios reconocen cuando están frente a un contenido realizado por una marca y valoran positivamente 
cuando sienten que se ha creado algo para ellos con un objetivo distinto al querer venderles y, además, sienten un compromiso y preocupación real de la marca con respecto a sus intereses y preferencias (Niño et al., 2016; Dans, 2017) reforzando el vínculo de confianza entre marca y usuario.

Desde que Balasubramanian (1994) estudió el carácter persuasivo de los formatos híbridos (aunque la comunicación por medio del branded content no utilice códigos o formatos comerciales, su causa primera no deja de tener un fin empresarial), estos han ido en expansión debido al desarrollo de nuevas tecnologías en comunicación. Hoy en día, es más preciso diferenciarlos por la tipología del contenido: custom publishing, advergaming, content marketing, branded entertainment, entre otros; siempre y cuando estén al servicio de los intangibles de la marca y el desarrollo de una experiencia positiva a través de un contenido valioso para el consumidor (López-Rúa, 2016).

\subsection{Branded content en redes sociales para la gestión de marcas universitarias}

Si las universidades desean gestionar su marca universitaria en redes sociales a partir de contenidos que les interesen a sus usuarios, deben conocer cómo se comportan en redes sociales. Las redes sociales no solo deben significar para ellas unas plataformas en donde pueden colocar información, debido a que una propuesta de comunicación unidireccional y centrada en la institución no genera resultados positivos para la marca (Rivera-Rogel et al., 2019). Capriotti (2009) señala que, para construir la imagen corporativa de la organización en la mente de los públicos, se debe trabajar a través de las diversas informaciones que han ido recibiendo de ella para formar una idea global. En este sentido, los espacios corporativos digitales de las universidades son lo que Britt (1971) catalogó como fuentes de información de imagen corporativa. Los stakeholders forman en sus mentes una idea o imagen de la organización después de haber tenido contacto con algunas de sus fuentes de información, desarrollando determinadas asociaciones cognitivas y emotivas.

El branded content es definido en la literatura académica de manera general como aquel contenido producido y planificado por una marca que aporta valor a los usuarios a través de la utilidad o el entretenimiento (Moyano et al., 2016), su valor depende del espacio comunicativo en donde se comparte con los consumidores. En las redes sociales, al momento de gestionar una marca universitaria, las universidades deben tener en cuenta, de manera especial, la dimensión socializadora. Ejemplo de ello son aquellos contenidos que las personas comparten para expresar su orgullo por algún logro personal o de la comunidad a la que pertenecen.

Coto y Borowiecka (2014) señalan cuatro elementos claves en el éxito de la comunicación social media: el contenido, el lenguaje, el auditorio y el motivo. El contenido responde a la realidad sobre la que se desea generar una conversación. El nivel de interacción sobre el contenido compartido dependerá de lo atractivo, útil e interesante que resulte para una determinada comunidad. El lenguaje hace referencia a los tonos y estilos de la comunicación, y deben corresponderse con la personalidad de la organización y las características de la comunidad. El auditorio consiste en tener en cuenta los intereses y preferencias de los públicos con los que se desea conectar para incentivar su interacción con los contenidos. El motivo responde a la razón por la cual buscamos la participación de la comunidad sobre el contenido compartido. Definir cuáles son los objetivos que se desean alcanzar con el contenido que se comparte, responde a una estrategia de la comunicación para establecer, fortalecer y mantener relaciones con las comunidades que aportarán valor a la organización.

En atención a lo expuesto hasta el momento, este estudio propone cuatro componentes o elementos comunicativos que pueden ser útiles para producir contenidos de valor en una estrategia de branded content en redes sociales para la gestión de la marca universitaria: la acción comunicativa, la temática del contenido, el formato y el vínculo emocional. Esta propuesta es uno de los aportes de la investigación y se sustenta en los trabajos realizados por Coto y Borowiecka (2014), Rivera-Rogel 
et al. (2019), Moyano et al. (2016), Coll (2019), Del Pino y Castelló-Martínez (2015) y Ashley y Tuten (2015). A continuación, se explican en la tabla 1.

Tabla 1. Elementos comunicativos en una estrategia de branded content

\begin{tabular}{|l|l|}
\hline Acción comunicativa & $\begin{array}{l}\text { En función al modo en que se desea generar valor para el usuario, la acción comu- } \\
\text { nicativa se refiere al modo en que se estructura el discurso de un contenido. Es el } \\
\text { elemento que guía el tono y estilo con el que se produce la publicación. Este puede } \\
\text { ser para informar, entretener, educar o inspirar al usuario. }\end{array}$ \\
\hline Temática & $\begin{array}{l}\text { Responde a la realidad sobre la que se desea generar interacción con el usuario. } \\
\text { Los temas deben guardar correspondencia con los intereses y necesidades de los } \\
\text { públicos, pero, al mismo tiempo, debe corresponder a los objetivos e identidad de la } \\
\text { organización. Consiste en equilibrar lo que la comunidad quiere escuchar y la organi- } \\
\text { zación quiere decir. }\end{array}$ \\
\hline Formato & $\begin{array}{l}\text { Implica abordar el contenido con una tipología y estilo de formato preferido por el } \\
\text { usuario en la red donde se comparte el contenido. Probablemente, una persona pre- } \\
\text { fiera leer un contenido sobre consejos de trabajo en Linkedln, y ver una infografía o } \\
\text { video que pueda compartir con sus amigos en Facebook. }\end{array}$ \\
\hline Vínculo emocional & $\begin{array}{l}\text { Se estructura el contenido con una emoción que sea capaz de suscitar el interés por } \\
\text { interactuar con la publicación. Incluso en los formatos informativos, existe un compo- } \\
\text { nente afectivo que motiva a los usuarios a interactuar con ellos. }\end{array}$ \\
\hline
\end{tabular}

Fuente: Elaboración propia a partir de los aportes de Coto y Borowiecka (2014), Rivera-Roguel et al. (2019), Moyano et al. (2016), Coll (2019), Del Pino y Castelló-Martínez (2015) y Ashley y Tuten (2015).

\section{Metodología para el análisis de los contenidos de las cuentas de Facebook de las universidades peruanas}

\subsection{Material}

Para la presente investigación, se ha seleccionado el ranking elaborado por la Sunedu debido a que considera indicadores de producción científica, los mismos que se toman como referencia del capital intelectual con el que cuentan las universidades peruanas, como sus profesores, investigaciones publicadas, proyectos de investigación. En los 10 primeros lugares del ranking presentado en el primer Informe bienal elaborado por la Sunedu en el 2018 figuran cinco universidades públicas, cuatro privadas asociativas y una universidad societaria. A continuación, se presenta la tabla 2 con el listado de las universidades.

Tabla 2. Top 10 de las mejores universidades peruanas

\begin{tabular}{|c|l|c|c|}
\hline Rank & \multicolumn{1}{|c|}{ Universidad } & Puntaje & Tipo \\
\hline 1 & Pontificia Universidad Católica del Perú & 100.000 & Asociativa \\
\hline 2 & Universidad Peruana Cayetano Heredia & 69.187 & Asociativa \\
\hline 3 & Universidad Nacional Mayor de San Marcos & 54.548 & Pública \\
\hline 4 & Universidad Nacional Agraria La Molina & 38.432 & Pública \\
\hline 5 & Universidad Nacional de Ingeniería & 26.086 & Pública \\
\hline 6 & Universidad Nacional de San Antonio Abad del Cusco & 23.584 & Pública \\
\hline 7 & Universidad Nacional de Trujillo & 20.045 & Pública \\
\hline 8 & Universidad Científica del Sur & 19.865 & Societaria \\
\hline 9 & Universidad de Piura & 14.843 & Asociativa \\
\hline 10 & Universidad del Pacífico & 11.088 & Asociativa \\
\hline
\end{tabular}

Fuente: Informe bienal sobre la realidad universitaria peruana según Sunedu publicado en el 2018. 
Con el objetivo de garantizar que el estudio aborde cuentas auténticas y gestionadas por la institución, se han seleccionado aquellas universidades que, estando en el top 10 del ranking de Sunedu, tengan la insignia de validación de la plataforma. Dentro del top 10 del ranking Sunedu, las universidades con una cuenta en Facebook verificada son la Universidad Católica del Perú, la Universidad del Pacífico, la Universidad Científica del Sur, la Universidad Nacional de Ingeniería y la Universidad de Piura.

Para este estudio, se aborda la red social Facebook por ser la más utilizada en el país. Según el estudio internacional Digital 2019 (We are social, 2019), 22 millones de peruanos utilizan redes sociales y el $100 \%$ de ellos utiliza Facebook. Además, el informe señala que, en ambos géneros, la mayor participación por edad promedio es entre los 18 y 34 años.

Tabla 3. Cuentas oficiales de Facebook de las universidades estudiadas

\begin{tabular}{|l|c|c|c|c|} 
Cuenta & $\begin{array}{l}\text { Número } \\
\text { de fans }\end{array}$ & $\begin{array}{l}\text { Número de } \\
\text { Publicaciones }\end{array}$ & $\begin{array}{l}\text { Total de } \\
\text { interacciones }\end{array}$ & $\begin{array}{l}\text { Engagement } \\
\text { rate }\end{array}$ \\
\hline @ucp & 503360 & 953 & 105286 & 20.9 \\
\hline @upacifico & 260015 & 450 & 75933 & 29.2 \\
\hline @universidadcientifica & 180787 & 39 & 7579 & 4.2 \\
\hline @UNloficial.pe & 112245 & 594 & 54366 & 48.4 \\
\hline @udepiura & 110343 & 458 & 42192 & 38.2 \\
\hline
\end{tabular}

Fuente: Elaboración propia en función a la información proporcionada por @Fanpage Karma.

\subsection{Método}

Con el objetivo de comprobar si las publicaciones desarrolladas en función a los lineamientos de branded content obtienen un mayor nivel de interacción, se ha desarrollado el estudio con una metodología de enfoque cuantitativo y cualitativo. El primero, a través de un análisis de contenido de enfoque cuantitativo (Andreu, 2002), permite medir los elementos comunicativos que alcanzaron un mejor rendimiento y, el segundo, desde una aproximación cualitativa, permite evaluar de qué modo estos elementos comunicativos están estructurados en las diez publicaciones que obtuvieron más interacciones (esta segunda aproximación sirve para enriquecer los hallazgos cuantitativos de la investigación y la discusión). A continuación, en la tabla 4, se presentan las variables y categorías que se han diseñado para realizar el análisis de contenido de las publicaciones seleccionadas.

Las variables responden a los elementos comunicativos, que son los componentes con los que se desarrollan las publicaciones para alcanzar los objetivos deseados. En el marco teórico del estudio, se han presentado los elementos comunicativos presentes en las publicaciones direccionadas a gestionar una marca universitaria en redes sociales a través de los lineamientos del branded content: acción comunicativa, formato del contenido, temática abordada y el vínculo emotivo. Así, se destacan los elementos que han generado mayor interacción. La finalidad es determinar los elementos que significan mayor valor para los usuarios. Las categorías se proponen después de haber realizado una primera aproximación al $20 \%$ de la muestra seleccionada. Tras verificar de qué modo se hacía visible y cuantificable el elemento comunicativo (expresado en la variable) se determinaron las categorías que integran la ficha de análisis de contenido. 
Tabla 4. Tabla de análisis de contenido para estudiar los elementos comunicativos

\begin{tabular}{|c|c|}
\hline Variable & Categoria \\
\hline \multirow{7}{*}{ Acción comunicativa } & Informar \\
\hline & Educar \\
\hline & Inspirar \\
\hline & Informar y educar \\
\hline & Informar e inspirar \\
\hline & Informar y convertir \\
\hline & Educar e inspirar \\
\hline \multirow{7}{*}{ Formato } & Videoblog \\
\hline & Video corporativo \\
\hline & Infografía \\
\hline & Fotografía \\
\hline & Blog (artículos, notas) \\
\hline & Video en vivo \\
\hline & Gráfica publicitaria \\
\hline \multirow{10}{*}{ Temática } & Comunicados institucionales \\
\hline & Logros institucionales \\
\hline & Coyuntura nacional \\
\hline & Valores y tendencias sociales \\
\hline & Valores e identidad institucional \\
\hline & Eventos y/o cursos \\
\hline & Trayectoria de egresados \\
\hline & Difusión de investigación e innovación científica \\
\hline & Novedades y tendencias del sector educación \\
\hline & Responsabilidad Social de la institución \\
\hline \multirow{7}{*}{ Vínculo emocional } & Orgullo \\
\hline & Preocupación \\
\hline & Deseo \\
\hline & Nostalgia \\
\hline & Curiosidad \\
\hline & Superación \\
\hline & Diversión \\
\hline
\end{tabular}

Fuente: Elaboración propia.

Para el análisis, se realizó la selección de 50 unidades de las cuentas de la PUCP, UP y UDEP. En el caso de la Universidad Científica del Sur, solo se seleccionaron 39 ya que no se contó con más publicaciones en el tiempo estudiado. En el caso de la UNI, solo 40 publicaciones cumplieron con los criterios de selección de la muestra (mayor número de interacciones recibido que consideran el número total de compartidos, comentarios y reacciones; vigencia del contenido en el momento del análisis; publicaciones que hayan sido realizadas con una descripción o copy; y contenidos de propia autoría ${ }^{3}$ ). En total, se seleccionaron 229 unidades de muestra para el análisis de los elementos comunicativos. Cabe destacar que, para la selección de la muestra, se ha empleado CFanpage Karma, un software especializado en Social Media Analytics con el cual se ha recogido un total de 2495 publicaciones realizadas en el periodo académico 2018-I.

\footnotetext{
${ }^{3}$ En ese sentido, no se han considerado enlaces de compartidos de otras cuentas o sitios web. Cuando las marcas comparten contenidos de autoría ajena se conoce como curación de contenidos (Coll, 2019).
} 


\section{Resultados}

Para poder comprender qué elementos comunicativos propician un mayor nivel de conexión con el público de las marcas universitarias en Facebook, a continuación, se presentan los resultados del análisis de los elementos comunicativos estudiados. Cada elemento será presentado en la tabla correspondiente con sus categorías de análisis. El total de interacciones de las publicaciones que corresponden a una misma categoría de análisis fueron sumadas y divididas entre el número de publicaciones para encontrar el promedio de interacción correspondiente.

Como se muestra a continuación, en la tabla 5, las publicaciones categorizadas con la acción comunicativa "informar" alcanzaron en promedio 768.1 interacciones, el nivel más bajo. Mientras que las publicaciones consideradas en la categoría "educar e inspirar" alcanzaron un nivel mayor de respuesta, obteniendo hasta 4317 interacciones por publicación.

Tabla 5. Nivel promedio de interacción alcanzado en las publicaciones por tipo de acción comunicativa

\begin{tabular}{|l|r|}
\hline Acción comunicativa & Promedio de interacción \\
\hline Informar & 768.1 \\
\hline Educar & 1032.9 \\
\hline Inspirar & 941.3 \\
\hline Informar y educar & 1457.0 \\
\hline Informar e inspirar & 851.6 \\
\hline Informar y convertir & 1180.5 \\
\hline Educar e inspirar & 4317.0 \\
\hline
\end{tabular}

Fuente: Elaboración propia con los datos extraídos de @FanpageKarma

Con respecto al elemento comunicativo referido a los formatos, se puede apreciar en la tabla 6 que el videoblog, de todos los formatos, fue el que mayor nivel de interacción en promedio alcanzó, con 2119.6 interacciones en promedio. El video corporativo fue el formato audiovisual que alcanzó, en segundo lugar, un mejor nivel de interacción. La infografía es el tercer formato con mayor nivel de interacción en promedio, con 1598.3 interacciones. En el caso de los videos en vivo o streaming, cabe resaltar que el nivel promedio de interacción alcanzado fue mayor que el de la gráfica publicitaria, que fue el más bajo de todos. Un ejemplo de video en vivo es el contenido llamado "Mesa UP", un programa en vivo que propicia una participación directa y en tiempo real de los usuarios durante la transmisión de un debate en el que profesores y colaboradores de la universidad discuten sobre un tema de interés público.

Tabla 6. Nivel promedio de interacción alcanzado en las publicaciones por tipo de formato

\begin{tabular}{|l|c|}
\hline Tipo de contenido & Promedio de interacción \\
\hline Videoblog & 2119.6 \\
\hline Video corporativo & 1649.4 \\
\hline Infografía & 1598.3 \\
\hline Fotografía & 1160.5 \\
\hline Blog (artículos, notas) & 841.1 \\
\hline Video en vivo & 718.0 \\
\hline Gráfica publicitaria & 193.4 \\
\hline
\end{tabular}

Fuente: Elaboración propia con los datos extraídos de @FanpageKarma 
En la medición realizada sobre los temas de mayor relevancia para los usuarios se encuentran, en primer lugar, aquellos contenidos sobre difusión de investigación y proyectos de innovación realizados por la institución, alcanzando 4232 interacciones en promedio por publicación. Por otro lado, dentro de las temáticas abordadas con nivel de interacción alto, destacan también las publicaciones relacionados con la trayectoria de egresados, los eventos institucionales, como ceremonias de graduaciones, y aquellas publicaciones para comunicar los valores e identidad de la institución. Con respecto a los contenidos que abordan temáticas relacionadas a la coyuntura nacional, y al sector de educación, la tabla 7 muestra que los usuarios participan con un nivel de interacción medio alto de los contenidos relacionados con su entorno.

Tabla 7. Nivel promedio de interacción alcanzado en las publicaciones por tipo de temática

\begin{tabular}{|l|c|}
\hline Temática del contenido & Promedio de interacción \\
\hline Comunicados institucionales & 544.4 \\
\hline Logros institucionales & 787.9 \\
\hline Coyuntura nacional & 1244.1 \\
\hline Valores y tendencias sociales & 486.6 \\
\hline Valores e identidad institucional & 1581.7 \\
\hline Eventos y/o cursos & 1315.7 \\
\hline Trayectoria de egresados & 1974.7 \\
\hline Difusión de investigación e innovación científica & 4232.3 \\
\hline Novedades y tendencias del sector educación & 1065.5 \\
\hline Responsabilidad Social de la institución & 389.0 \\
\hline
\end{tabular}

Fuente: Elaboración propia con los datos extraídos de @FanpageKarma

Los resultados de la tabla 8 muestran que las publicaciones vinculadas al orgullo institucional tienen una interacción muy elevada. Como señaló un estudio realizado por Peruta y Helm (2018), las universidades cuentan en sus cuentas oficiales con seguidores muy vinculados emocionalmente a sus noticias de investigación, triunfos deportivos y logros universitarios. Por otro lado, si bien los resultados muestran un nivel de interacción muy parejo entre los tipos de vínculos emocionales gestionados, destaca también la preocupación, especialmente, cuando es gestionada en función a los problemas del entorno de los usuarios.

Tabla 8. Nivel promedio de interacción alcanzado en las publicaciones por tipo de vínculo emocional

\begin{tabular}{|l|c|}
\hline Emoción & Promedio de interacción \\
\hline Orgullo & 1313.4 \\
\hline Preocupación & 1010.4 \\
\hline Deseo & 788.8 \\
\hline Nostalgia & 1097.9 \\
\hline Curiosidad & 943.8 \\
\hline Superación & 726.8 \\
\hline Diversión & 336.6 \\
\hline
\end{tabular}

Fuente: Elaboración propia con los datos extraídos de @FanpageKarma 


\section{Conclusiones y Discusión}

Las universidades peruanas estudiadas utilizan sus cuentas oficiales de Facebook para acciones de comunicación diversas y variadas, como la difusión de investigaciones y de noticias institucionales, invitaciones a eventos, actividades para estudiantes y profesores, comunicados institucionales, anuncios de carreras y especialidades, eventos de graduados, entre otras. A nivel de mensaje, en todos los casos estudiados, el discurso de la marca no se alinea a una estrategia de posicionamiento sostenida, sino a mensajes reactivos y espontáneos relacionados con el quehacer de la universidad o a los sucesos del entorno en los que participa. Esto significa que las publicaciones no presentan un orden o una pauta que responda a un posible plan en la generación de contenido para esta red social.

Los resultados de la investigación muestran que aquellos contenidos con una acción comunicativa que se inspira a través del contenido educativo alcanzan un mayor nivel de interacción promedio. Además, se encuentra una mayor participación de los usuarios en publicaciones vinculadas a la emoción del orgullo institucional, preocupación por la sociedad (desarrollados en formatos audiovisuales e interactivos).

Dentro de los contenidos con más nivel de interacción, se encontró un alto nivel de interacción en contenidos relacionados con temas de política, como casos de corrupción en el Perú, y a elementos del entorno de interés nacional en general. Asimismo, cabe señalar que la temática abordada que registró el nivel de interacción más alto fue la relacionada a comunicar el desarrollo de un proyecto de investigación e innovación científica vinculado a un importante aporte a la sociedad. En este tipo de contenidos el aporte se da a través de las enseñanzas o consejos útiles que deja el proyecto, y no solo la información de su realización. Este resultado demuestra la relevancia que ha adquirido la comunicación de la investigación para la reputación de las universidades. Gómez y Paniagua (2014) precisaban, para el caso de Twitter, que existía poca atención a la actividad investigadora. En esta investigación, se pone en evidencia que las universidades peruanas sí publican contenido sobre investigación e innovación en sus cuentas oficiales de Facebook.

Estas cuentas no son espacios de comunicación al servicio del entretenimiento y el comercio digital. Por el contrario, las estructuras de comunicación responden a una propuesta informativa y educativa. Al ser las universidades un referente social que alcanzan orientación confiable sobre los temas del entorno (Villegas, 2018), se encuentra una mayor colaboración de los profesores para generar contenido sobre temas que interesen a los públicos en un momento determinado. Sin embargo, debido a que ese interés responde también a un tema de coyuntura, es difícil estructurar para una universidad una estrategia con una programación de largo plazo. En ese caso, resulta preferible desarrollar una propuesta de branded content con una estructura de temas flexibles en redes sociales, en donde se aproveche el capital intelectual de las universidades para generar propuestas de valor.

En esta investigación se ha puesto especial atención en el indicador de interacción debido a su importancia en la nueva economía digital, en la que se valora el crecimiento de la comunidad de usuarios a través de la creación de contenidos que generan una alta respuesta (Coll, 2019). En el caso de las cuentas oficiales de Facebook de las universidades estudiadas, se encontró que las publicaciones de mayor nivel de interacción son las que gestionan la emoción del orgullo de marca y aportan una formación de interés a través de historias que educan e inspiran. Ante lo expuesto, la hipótesis "en las cuentas oficiales de Facebook de las mejores universidades del Perú, según la Superintendencia Nacional de Educación Superior Universitaria (Sunedu), las publicaciones con mayor interacción son aquellas que aportan un contenido que educa e inspira a partir del capital intelectual con el que cuentan las instituciones universitarias (investigaciones), usando formatos audiovisuales y apelando al orgullo que puede despertar en su comunidad dichas publicaciones" se comprueba. 
Este estudio plantea que las universidades contemplen el branded content, más que como una estrategia específica de comunicación para producir determinados contenidos, como una filosofía que guíe la gestión de sus estrategias en redes sociales. La idea es desarrollar los contenidos con un enfoque que se centre en dar un aporte de interés a sus públicos, al mismo tiempo que asocie un aspecto de la institución con el orgullo y prestigio de la marca. De ese modo, podrán generar un nivel de participación e interactividad real con sus públicos y lograr acercar a los usuarios al conocimiento de su accionar, eventos relevantes, labores de responsabilidad social y desarrollo de la investigación (Puertas-Hidalgo y Carpio-Jiménez, 2017). Esto último es importante ya que, como señalan Del Pino y Castelló-Martínez (2015), aunque el desarrollo de contenidos guiados por los lineamentos del branded content se planteen con el objetivo de sorprender al usuario, su realización siempre está guiada en base a los intereses de la institución. En ese sentido, aun en los casos en donde la marca es protagonista del contenido, su nivel de interacción dependerá de que los elementos comunicativos utilizados sean afines a los intereses y gustos de los usuarios y al empleo de recursos narrativos propios del branded content, los cuales poseen un nivel persuasivo que maximiza las posibilidades de conectar con las personas (Atarama-Rojas y Sánchez, 2018).

Como se ha mencionado, el modo de materializar esa propuesta de valor puede ser variada en su tipología. Decidir la producción del contenido adecuado para el desarrollo de una estrategia de branded content dependerá de los estilos y modos que el usuario prefiere cuando consume un contenido, y de las expectativas con las que utiliza determinada plataforma comunicativa. En el caso de las universidades, el proceso es más complicado porque deben responder a los gustos e intereses de distintos públicos (Rivera-Rogel et al., 2019). En este sentido, es necesario el desarrollo de una auditoría de redes que ayude a identificar los elementos a los que mejor responden los usuarios (Opresnik, 2018). En esta línea, las oficinas de comunicación de las universidades deben estar atentas a qué fórmulas y propuestas de contenido significan mayor valor e interés para sus usuarios. El único modo de lograrlo es creando contenidos, midiéndolos y aprendiendo de los resultados. En este proceso, son claves la experimentación y el aprendizaje constante en la planificación estratégica de contenidos, conocida como growth hacking, para el desarrollo de estrategias en redes sociales al servicio de las relaciones y el crecimiento acelerado de comunidades en red (Coll, 2019).

Las universidades estudiadas disponen de un capital intelectual importante para ser utilizado de manera estratégica en la producción de contenido que aporte utilidad al usuario, y que, al mismo tiempo, incremente su visibilidad y prestigio institucional. Aprovecharlo, exige que los departamentos de comunicación estén atentos a los intereses de los usuarios y a su entorno, y que cuenten con una estructura institucional caracterizada por la participación de sus actores, principalmente, de estudiantes y profesores, quienes son los que concretizan y proyectan la misión y visión de la marca universitaria, además de potenciarla de un aspecto socializador que contribuye a un mayor crecimiento de su comunidad digital.

\section{Bibliografía}

Andreu, J. (2002). Las técnicas del análisis de contenido: una revisión actualizada. Fundación Centro de Estudios Andaluces.

Ashley, C., y Tuten, T. (2015). Creative strategies in social media marketing: An exploratory study of branded social content and consumer engagement. Psychology \& Marketing, 32(1), 15-27. https://doi.org/10.1002/mar.20761

Atarama, T. y Cortéz, C. (2015). La gestión de la reputación digital en las universidades: Twitter como herramienta de la comunicación reputacional en las universidades peruanas. Revista de comunicación, (14), 26-47. https://revistadecomunicacion.com/es/articulos/2015/Art026-047.html 
Atarama-Rojas, T. y Sánchez, C. (2018). Storytelling corporativo y responsabilidad social corporativa: análisis del caso "The promise" de Volvo. Organicom, 14(27), 124-133. https://doi. org/10.11606/issn.2238-2593.organicom.2017.144114

Balasubramanian, S. (1994). Beyond Advertising and Publicity: Hybrid Messages and Public Policy issues. Journal of Advertising, 23(4), 29-46. https://doi.org/10.1080/00913367.1943.10673 457

Britt, S. H. (1971). Psychological principles of the corporate imagery mix. Business Horizons, 14(3), 55-59. https://doi.org/10.1016/0007-6813(71)90118-2

Coll, P. (2019). El marketing de contenidos en la estrategia de growth hacking en la nueva economía. Los casos de Wallapop, Westwing y Fotocasa. adComunica. Revista Cientifica de Estrategias, Tendencias e Innovación en Comunicación, (17), 105-116. https://doi. org/10.6035/2174-0992.2019.17.7

Coto, A. y Borowiecka, M. (2014). El plan de social media marketing. Pearson.

Critikián, D. M. (2017). Cómo valoran y emplean los alumnos de la Universidad Camilo José Cela el Facebook de su universidad. En Uso y aplicación de las redes sociales en el mundo audiovisual y publicitario (pp. 7-16). McGraw-Hill.

Curiel, C. y Ferreira, L. (2017). Comunicación y Social Media en las Empresas de Moda. Revista Prisma Social, (18), 226-258. https://goo.gl/9BYNTK

Dans, A. (2017). Informa D\&B: Redefiniendo el branded content. En Comunicación y estrategia. Casos prácticos en gestión de la reputación. Pearson.

Del Pino, C. y Castelló-Martínez, A. (2015). La comunicación publicitaria se pone de moda: branded content y fashion films. Revista Mediterránea de Comunicación, 6(1), 105-128. https:// doi.org/10.14198/MEDCOM2015.6.1.07.

García-Feijoo, M.; Eyzaguirre, A. y Yabar, J. (2016). La página web como herramienta de promoción/educación en un programa universitario presencial. En Camarero, C. y Rodríguez, T. (2016). Estrategias en comunicación y su evolución en los discursos (pp. 187-198). McGraw-Hill.

Gómez, B. y Paniagua, F. (2014). Las universidades españolas en Twitter: Mensajes, contenidos y públicos. Historia y Comunicación Social, 19(Esp. Enero), 681-694. https://doi.org/10.5209/ rev_HICS.2014.v19.44994

Koivula, A.; Keipi, T.; Koiranen, I. y Räsänen, P. (2018). A Middle-Aged Social Internet with a Millennial Exodus? Changes in Identifications with Online Communities Between 2009 and 2017 in Finland. En Social Computing and Social Media. User Experience and Behavior. Springer, Cham. https://doi.org/10.1007/978-3-319-91521-0_23

López-Rúa, M. (2016). Content Marketing: Nueva vía de comunicación persuasiva. En Camarero, C. y Rodríguez, T. (2016). Estrategias en comunicación y su evolución en los discursos (pp. 187198). McGraw-Hill.

Mazza, B. y Palermo A. (2019). Creation of Social Media Content and the Business Dialogic Process. En Túñez-López M., Martínez-Fernández VA., López-García X., Rúas-Araújo X., Campos-Freire F. (eds). Communication: Innovation \& Quality. Studies in Systems, Decision and Control. Springer, Cham. https://doi.org/10.1007/978-3-319-91860-0_22 
Moyano, J., Baños, G., y Ramírez, F. (2016). Los Mensajes Híbridos en el marketing postmoderno: una propuesta de taxonomía. ICONO14, Revista científica de Comunicación y Tecnologías emergentes, 14(1), 26-57. https://doi.org/10.7195/ri14.v14i1.890

Mosquera, M. y Bartolomé, A. (2014): Redes sociales y consumidores: participación y construcción de imagen. Historia y Comunicación Social, 19(Especial Febrero), 211-221. https:// doi.org/ 10.5209/rev_HICS.2014.v19.45022

Niño, J.; González, J. y Valderrama, M. (2016). El futuro de la publicidad digital: el obligado impulso de las redes sociales. En Camarero, C. y Rodríguez, T. (2016). Estrategias en comunicación y su evolución en los discursos (pp. 187-198). McGraw-Hill.

Opresnik, M.O. (2018). Effective Social Media Marketing Planning - How to Develop a Digital Marketing Plan. En Meiselwitz G. (eds). Social Computing and Social Media. User Experience and Behavior. Springer, Cham. https://doi.org/10.1007/978-3-319-91521-0_24

Parejo, M.; Martín-Pena, D. y Pinto-Zúñiga, R. (2016). El nuevo rol de las universidades en la comunicación científica. Actas del I Congreso Internacional Comunicación y Pensamiento. Comunicracia y desarrollo social, 523-539. https://goo.gl/WZqHKL

Peruta, A. y Helm, C. (2018). Engaging University Alumni Through Social Media: Strategies for Creating Community. The Journal of Social Media in Society Spring, 7(1), 123-150. Recuperado de http://thejsms.org/tsmri/index.php/TSMRI/article/view/222/156

Pineda, A. (2009). Branded content antes del branded content: la Modelación Cultural Propagandística como forma de propaganda encubierta. Ámbitos, (18), 117-134. https://goo.gl/Bb4rFU

Puertas-Hidalgo, R. y Carpio-Jiménez, L. (2017). Interacción generada en redes sociales por las universidades categoría A en Ecuador. International Journal of Information Systems and Software Engineering for Big Companies, 4(1), 53-61. https://goo.g1/Rp9phR

Ramos, M. (2006). Cuando la marca ofrece entretenimiento: aproximación al concepto de advertainment. Questiones publicitarias, 1(11), 33-49. https://doi.org/10.5565/rev/qp.134

Reina, J., Fernández, I., y Noguer, Á. (2012). El uso de las Redes sociales en las Universidades andaluzas: el caso de Facebook y Twitter. Revista Internacional de Relaciones Públicas, 2(4), 123-144. https://doi.org/10.5783/RIRP-4-2012-06-123-144

Rivera-Rogel, D.; Yaguache, J., Velásquez, A. y Paladines, F. (2019). Social Networks as a New University Venue. En Túñez-López M., Martínez-Fernández VA., López-García X., Rúas-Araújo X., Campos-Freire F. (eds). Communication: Innovation \& Quality. Studies in Systems, Decision and Control. Springer, Cham. https://doi.org/ 10.1007/978-3-319-91860-0_30

Rodrigo, E.; García, S. y Martín, S. (2011). El complejo mundo de la interactividad: emociones y redes sociales. Revista Mediterránea de Comunicación, 2(1), 171-190. https://doi.org/ 10.14198/ MEDCOM2011.2.10

Rodríguez-Fernández, M., Sánchez-Amboage, E. y Juanatey-Boga, O. (2019). Virtual Tourist Communities and Online Travel Communities. En Túñez-López M., Martínez-Fernández VA., López-García X., Rúas-Araújo X., Campos-Freire F. (eds). Communication: Innovation \& Quality. Studies in Systems, Decision and Control. Springer, Cham. https://doi.org/10.1007/978-3319-91860-0_26

Sáez, J., Núñez, M. y Cerdá, E. (2016). Rock\&Love en la comunicación publicitaria en la era digital: un análisis de la creatividad de las marcas entre el 2011 y el 2015. En Camarero, C. y 
Rodríguez, T. (2016). Estrategias en comunicación y su evolución en los discursos (pp. 187-198). McGraw-Hill.

Serrano-Puche, J. (2016). Internet y emociones: nuevas tendencias en un campo de investigación emergente. Comunicar, 24(46), 19-26. https://doi.org/10.3916/C46-2016-02

Sunedu (2018). Informe bienal sobre la realidad universitaria peruana. https://www.sunedu.gob. pe/informe-bienal-sobre-realidad-universitaria/

Sunedu (2019). Historia. https://www.sunedu.gob.pe/historia/

Túñez-López M.; Valarezo-González K. y Punín-Larrea, M. (2019). Generation Co. Tendencies of XXI Century's Communication Management. En Túñez-López M., Martínez-Fernández VA., López-García X., Rúas-Araújo X., Campos-Freire F. (eds). Communication: Innovation \& Quality. Studies in Systems, Decision and Control. Springer, Cham. https://doi.org/10.1007/9783-319-91860-0_21

Villegas, A. Q. (2018). Responsabilidad Social Universitaria. Big Bang Faustiniano.

We are social. (2019). Digital 2019: Global Internet Use Accelerates. https://wearesocial.com/us/ blog/2019/01/digital-2019-global-internet-use-accelerates 